\title{
Behavioural therapies versus treatment as usual for depression
}

\author{
Deborah Caldwell ${ }^{1}$, Vivien Hunot ${ }^{2}$, Theresa HM Moore ${ }^{2}$, Philippa Davies ${ }^{2}$, Hannah Jones ${ }^{3}$, \\ Glyn Lewis ${ }^{1}$, and Rachel Churchill ${ }^{2}$ \\ ${ }^{1}$ School of Social and Community Medicine, University of Bristol, Bristol, UK \\ ${ }^{2}$ Academic Unit of Psychiatry, School of Social and Community Medicine, University of Bristol, \\ Bristol, UK \\ ${ }^{3}$ Cochrane Schizophrenia Group, The University of Nottingham, Nottingham, UK
}

\begin{abstract}
This is the protocol for a review and there is no abstract. The objectives are as follows:

1. To examine the effectiveness and acceptability of all $\mathrm{BT}$ approaches compared with treatment as usual/waiting list/attention placebo control conditions for acute depression.

2. To examine the effectiveness and acceptability of different BT approaches (behavioural therapy, behavioural activation, social skills training and relaxation training) compared with treatment as usual/waiting list/attention placebo control conditions for acute depression.
\end{abstract}

3. To examine the effectiveness and acceptability of all BT approaches compared with different types of comparator (standard care, no treatment, waiting list, attention placebo) for acute depression.

Copyright (C) 2012 The Cochrane Collaboration. Published by John Wiley \& Sons, Ltd.

Contact address: Rachel Churchill, Academic Unit of Psychiatry, School of Social and Community Medicine, University of Bristol, Oakfield House, Oakfield Grove, Bristol, Avon, BS8 2BN, UK. rachel.churchill@ccdan.org. rachel.churchill@bristol.ac.uk.

Editorial group: Cochrane Depression, Anxiety and Neurosis Group.

Publication status and date: Edited (no change to conclusions), published in Issue 7, 2012.

CONTRIBUTIONS OF AUTHORS

Deborah Caldwell provided methodological and statistical advice for each of 12 psychotherapies for depression protocols. She contributed to the design of the data extraction form, drafted sections of the protocols and commented on the protocol manuscripts. She is responsible for the preparation and writing of the review. She designed the plan for the multiple treatment meta-analysis for the overview of reviews.

Vivien Hunot provided theoretical and clinical expertise for designing this programme of linked reviews, drawing from her training and clinical practice as a psychotherapeutic counsellor and cognitive behavioural therapist in NHS primary care settings. She worked on protocol development, developing a search strategy and compiling data extraction forms, and wrote the protocols for each review. Along with Dr Rachel Churchill, she conducted the original review on which this programme is based.

Theresa Moore is managing the organisation of data for the 12 linked reviews of psychotherapies for depression including the search results, tracking of papers, and management of references for the project. She has developed the data collection forms. She designed the database and spreadsheets for data collection and has contributed to writing sections of the protocols and commented on text of the protocols.

Philippa Davies contributed to the design of the review and development of the protocol.

Glyn Lewis provided a clinical perspective on 12 linked psychotherapies for depression protocols.

Rachel Churchill conceived, designed, secured funding for, and is managing this programme of linked reviews. She has worked on all aspects of the development of this project, including building a review team, protocol development, developing a search strategy and compiling data-extraction forms. Along with Dr Vivien Hunot, she conducted the original review on which this programme is based. She is guarantor of the individual reviews in this programme of work.

Hannah Jones read and commented on the protocols.

DECLARATIONS OF INTEREST

None known. 


\section{BACKGROUND}

\section{Description of the condition}

Major depression is characterised by persistent low mood and loss of interest in pleasurable activities, accompanied by a range of symptoms including weight loss, insomnia, fatigue, loss of energy, inappropriate guilt, poor concentration and morbid thoughts of death (APA 2000). Somatic complaints are also a common feature of depression, and people with severe depression may develop psychotic symptoms (APA 2000).

Depression is the third leading cause of disease burden world-wide and is expected to show a rising trend over the next 20 years (WHO 2004; WHO 2008). A recent European study has estimated the point prevalence of major depression and dysthymia at $3.9 \%$ and $1.1 \%$ respectively (ESEMeD/MHEDEA 2004). As the largest source of non-fatal disease burden in the world, accounting for $12 \%$ of years lived with disability (Ustun 2004), depression is associated with marked personal, social and economic morbidity, loss of functioning and productivity and creates significant demands on service providers in terms of workload (NICE 2009). Depression is also associated with a significantly increased risk of mortality (Cuijpers 2002). The strength of this association, even taking account of confounders such as physical impairment, health-related behaviours and socio-economic factors, has been shown to be comparable to, or greater than, the strength of the association between smoking and mortality (Mykletun 2009).

\section{Description of the intervention}

Clinical guidelines recommend pharmacological and psychological interventions, alone or in combination, in the treatment of moderate to severe depression (NICE 2009). The prescribing of antidepressants has increased dramatically in many Western countries over the last 20 years, mainly with the advent of selective serotonin reuptake inhibitors and newer agents such as venlafaxine. Antidepressants remain the mainstay of treatment for depression in health care settings (Ellis 2004; NICE 2009).

Whilst antidepressants are of proven efficacy for the acute treatment of depression (Cipriani 2005; Guaiana 2007; Arroll 2009; Cipriani 2009; Cipriani 2009a; Cipriani 2009b), adherence rates remain very low (Hunot 2007; van Geffen 2009), in part due to patients' concerns about side effects and possible dependency (Hunot 2007). Furthermore, surveys consistently demonstrate patients' preference for psychological therapies over that of antidepressants (Churchill 2000; Riedel-Heller 2005). Therefore, psychological therapies provide an important alternative or adjunctive intervention for depressive disorders.

A diverse range of psychological therapies is now available for the treatment of common mental disorders (Pilgrim 2002). Psychological therapies may be broadly categorised into four separate philosophical and theoretical schools, comprising psychoanalytic/dynamic (Freud 1949; Klein 1960; Jung 1963), behavioural (Watson 1924; Skinner 1953; Wolpe 1958), humanistic (Maslow 1943; Rogers 1951; May 1961) and cognitive approaches (Lazarus 1971; Beck 1979). Each of these four schools incorporates a number of differing and overlapping psychotherapeutic approaches. Some psychotherapeutic approaches, such 
as cognitive analytic therapy (Ryle 1990), explicitly integrate components from several theoretical schools. Other approaches, such as interpersonal therapy for depression (Klerman 1984), have been developed to address characteristics considered to be specific to the disorder of interest.

Behaviour therapy became a dominant force in the 1950s, drawing from the work of Skinner 1953, Wolpe 1958 and Eysenck 1960. Behaviour therapy (BT) emphasises the role of environmental cues in influencing the acquisition and maintenance of behaviour (Nelson Jones 1990), and in contrast with psychoanalysis, was developed through experimentally derived principles of learning (Rachman 1997).

A number of BT models have been developed for the treatment of depression, including behavioural activation (BA) (Jacobson 1996), social skills training (Bellack 1980) and Lewinsohn's behavioural therapy approach (Lewinsohn 1974). Some models initially developed as behavioural treatments, including problem-solving therapy (Nezu 1986), selfcontrol therapy (Fuchs 1977; Rehm 1977) and the Coping with Depression program (Lewinsohn 1984) have, over time, integrated cognitive techniques (Jacobson 2001) (see Types of interventions section for a description of these approaches).

\section{How the intervention might work}

Skinner 1953 proposed that depression was associated with an interruption of established sequences of healthy behaviour that were previously positively reinforced by the social environment, based on operant conditioning principles (in which behaviour patterns are learnt, rather than instinctive). In subsequent expansions of this model, reduction of positively reinforced healthy behaviour has also been attributed to a decrease in the number and range of reinforcing stimuli available to the individual, a lack of skill in obtaining positive reinforcement (Lewinsohn 1974) and/or increased frequency of punishment (Lewinsohn 1984).

Conventional BT models for depression focus attention on increasing access to pleasant events and positive reinforcers and decreasing the intensity and frequency of events and consequences deemed to be unpleasant/negative (Lewinsohn 1972), through monitoring of pleasant events, activity scheduling, social skills development, assertiveness training, relaxation therapy and time management training (Hopko 2003).

\section{Why it is important to do this review}

With the advent of cognitive therapy in the 1970s, BT approaches based on purely operant and respondent principles came to be regarded as insufficient. However, in the past 10 to15 years, there has been renewed interest in the feasibility of behavioural treatments for depression (Hopko 2003). A recent meta-analysis of 17 RCTs comparing BT against controls or other psychotherapies suggested superior outcomes compared with comparators in terms of depression recovery rates, symptom levels and participant dropout (Ekers 2008). The NICE 2009 guidelines recommend the use of behavioural activation (BA) for moderate to severe depressive disorder, whilst acknowledging that the evidence for BA is currently less robust. However, in this and other recent systematic reviews, the inclusion of a heterogeneous group of studies and studies using an 'extended' behavioural activation 
approach (regarded as a 'third wave' CBT intervention) limits interpretation of the findings (Cuijpers 2007; Ekers 2008).

Given the resurgence of interest in the use of BT as a cost-effective intervention for depression which is simple to deliver, a comprehensive review of the comparative effectiveness and acceptability of BT interventions for depression is now timely to inform and update clinical practice and future clinical guideline development. This review forms part of a programme of 12 reviews covering behavioural, cognitive behavioural, psychodynamic, interpersonal, cognitive analytic and other integrative, humanistic and mindfulness-based 'third wave' cognitive and behavioural psychological therapies, all compared with treatment as usual or with one another.

\section{OBJECTIVES}

1. To examine the effectiveness and acceptability of all BT approaches compared with treatment as usual/waiting list/attention placebo control conditions for acute depression.

2. To examine the effectiveness and acceptability of different BT approaches (behavioural therapy, behavioural activation, social skills training and relaxation training) compared with treatment as usual/waiting list/attention placebo control conditions for acute depression.

3. To examine the effectiveness and acceptability of all BT approaches compared with different types of comparator (standard care, no treatment, waiting list, attention placebo) for acute depression.

\section{METHODS}

\section{Criteria for considering studies for this review}

Types of studies-Randomised controlled trials (RCTs) will be eligible for inclusion in the review. Trials employing a cross-over design will be included in the review (whilst acknowledging that this design is rarely used in psychological therapy trials) but data from the first active treatment phase only will be used. Cluster RCTs will also be eligible for inclusion.

Quasi-randomised controlled trials, in which treatment assignment is decided through methods such as alternate days of the week, will not be eligible for inclusion.

\section{Types of participants}

Participant characteristics: Studies of men and women aged 18 years will be included. A Cochrane review on psychotherapy for depression in children and adolescents ( $<18$ years) has been undertaken separately and is soon to be published (Watanabe 2004). The increasing prevalence of memory decline (Ivnik 1992), cognitive impairment (Rait 2005) and multiple comorbid physical disorders/polypharmacy (Chen 2001) in individuals over 74 years may differentially influence the process and effect of psychological therapy interventions. Therefore, to ensure that older patients are appropriately represented in the review (Bayer 
2000; McMurdo 2005), an upper age cut-off of $<75$ years will be used (when a study may have included individuals 75 , we will include it so long as the average age is $<75$ ) and a previously published Cochrane review on psychotherapeutic treatments for older depressed people (Wilson 2008) will be updated concurrently by the authors.

Setting: Studies may be conducted in a primary, secondary or community setting, and will include volunteers. Studies involving inpatients will be excluded. Studies that focus on specific populations - nurses, care givers, depressed participants at a specific work place will be included if the participants all meet the criteria for depression.

Diagnosis: We will include all studies that focus on acute phase treatment of clinically diagnosed depression.

1. Studies adopting any standardised diagnostic criteria to define participants suffering from an acute phase unipolar depressive disorder will be included. Accepted diagnostic criteria include Feighner criteria, Research Diagnostic Criteria, DSM-III (APA 1980), DSM-III-R (APA 1987), DSM-IV-TR (APA 2000) or ICD-10 (WHO 1992) criteria. Earlier studies may have used ICD-9 (WHO 1978), but ICD-9 is not based on operationalised criteria, so studies using ICD-9 will be excluded from this category.

2. Mild, moderate and severe depressive disorders are all found in primary care (Mitchell 2009; Rait 2009; Roca 2009). In order to fully represent the broad spectrum of severity of depressive symptoms encountered by healthcare professionals in primary care, studies that used non-operationalised diagnostic criteria or used a validated clinician or self-report depression symptom questionnaire, such as Hamilton Rating Scale for Depression (Hamilton 1960) and Beck Depression Inventory (Beck 1961), to identify depression caseness based on a recognised threshold, will also included. However, the influence of including this category of studies will be examined in a sensitivity analysis.

Studies focusing on chronic depression or treatment resistant depression, i.e. studies that list these conditions as inclusion criteria, will be excluded from the review. Studies in which participants are receiving treatment to prevent relapse following a depressive episode (that is, where participants are not depressed at study entry) will also be excluded.

Studies of people described as 'at risk of suicide', or with dysthymia or other affective disorders such as panic disorder will be included if the participants meet criteria for depression as stated above, but will be excluded if not.

We will not include subgroup analyses of people with depression, selected out of people with mixed diagnoses, because such studies would be susceptible to publication bias (the authors reported such subgroup studies because the results were "interesting"). In other words we will include such studies only if the inclusion criteria for the entire study satisfied our eligibility criteria.

Comorbidity: Studies involving participants with comorbid physical or common mental disorders will be eligible for inclusion, as long as the comorbidity is not the focus of the 
study. In other words, we will exclude such studies which focused on depression among patients with Parkinson's disease or after acute myocardial infarction but will accept such studies which may have included some subjects with Parkinson's disease or with acute myocardial infarction.

\section{Types of interventions}

Experimental interventions: BT approaches eligible for inclusion will be grouped into four main categories, according to the specific therapeutic principles and techniques described by trial authors, as follows:

1. Behavioural therapy: Lewinsohn 1974 proposed that depressed individuals have low rates of pleasant activities and obtained pleasure, that their mood covaries with rates of pleasant and aversive activities, that their mood improves with increases in pleasant activities, and that they lack social skills during the depressed phase. Therefore, behavioural therapy based on the approach developed by Lewinsohn and colleagues involves helping individuals increase their frequency and quality of pleasant activities, producing corresponding improvement in mood and overall quality of life (Lewinsohn 1974).

2. Behavioural activation (original model): The original model of behavioural activation (BA) developed by Jacobson 1996 was defined primarily by the proscription of cognitive interventions (Dimidjian 2006), and was tested in a dismantling study in which the behavioural activation component of cognitive therapy for depression was isolated (Beck 1979). Based on its original design, BA model components include increasing access to pleasant events and consequences, activity scheduling and social skills development, thereby helping people to make contact with potentially reinforcing experiences (Jacobson 2001). No attempt is made to directly restructure cognitions.

3. Social skills training/assertiveness training: The social skills training model (SST) proposes that depressed people may have difficulty initiating, maintaining and ending conversations (Jackson 1985). Because of these deficits, the individual is unable to elicit mutually reinforcing behaviour from other people in their environment. SST subsumes assertion and conversational skills, together with more specialised subskills such as dating and job interview skills. Four social contexts of interacting with strangers, friends, family members and people at work or school are targeted (Bellack 1980) and interventions such as instruction, modelling, rehearsal, feedback and reinforcement are used to enable the development of new responses (Jackson 1985).

As assertiveness training comprises a key component of SST, it will be included in the SST category.

4. Relaxation therapy: Relaxation Training is a behavioural stress management technique that induces a relaxation response, helping to switch off the fight/flight response and causing the levels of stress hormones in the blood stream to fall. There are a variety of techniques for inducing relaxation, the most common of which is Jacobson's progressive muscle relaxation training (Bernstein 1973). 
Other behavioural therapies: Where a study evaluating a behavioural therapy intervention not listed above is identified, a post-hoc decision will be made about its inclusion in the review, with the impact of its inclusion examined in a sensitivity analysis (see Methods section).

Comparators: The control comparison will be 'treatment as usual' (also called standard care, usual care or no treatment; TAU), waiting list (WL), attention placebo (AP) and psychological placebo (PP). The type of 'treatment as usual' comparator used is likely to influence the observed effectiveness of the intervention and, where possible, for the purposes of heterogeneity analyses, comparators will be subgrouped as waiting list, treatment as usual/usual care or attention placebo. In each study, descriptions of 'treatment as usual' and attention placebo conditions will be scrutinised to ensure that they do not comprise an active psychological therapy treatment. Within the 'treatment as usual' and waiting list conditions, participants may receive any appropriate medical care during the course of the study on a naturalistic basis, including pharmacotherapy and/or psychological therapy, as deemed necessary by the clinician. Additional treatment(s) received naturalistically by participants in both the control and active comparisons for each included study will be documented.

Format of psychological therapies: The psychological therapy intervention is required to be delivered through face to face meetings between the patient and therapist. Interventions in which face to face therapy is augmented by telephone or Internet-based support will be included in the review. Psychological therapy approaches conducted on either an individual or on a group basis will be eligible for inclusion. There is no limit to the number of sessions and we accept psychological therapy delivered in only one session.

Excluded interventions: The behavioural activation (BA) approach has been extended (Jacobson 2001; Martell 2001), by introducing contextual and idiosyncratic functional analysis in the assessment and treatment of depression. The extended BA approach is regarded as a third wave cognitive behavioural therapy and, therefore, for the purposes of this review will be categorised as a comparator third wave CBT intervention.

Counselling interventions traditionally draw from a wide range of psychological therapy models, including person-centred, psychodynamic and cognitive behavioural approaches, applied integratively, according to the theoretical orientation of practitioners (Stiles 2008). Therefore, studies of counselling will usually be included in the integrative therapies reviews. However if the counselling intervention consists of a single discrete psychological therapy approach, it will be categorised as such, even if the intervention is referred to as 'counselling'. If the intervention is manualised, this will inform our classification.

Psychological therapy models based on social constructionist principles (that focus on the ways in which individuals and groups participate in the construction of their perceived social reality) including couples therapy, family therapy, solution-focused therapy, narrative therapy, personal construct therapy, neuro-linguistic programming and brief problemsolving (Watzlavick 1974) will be excluded. These therapies work with patterns and dynamics of relating within and between family, social and cultural systems in order to 
create a socially constructed framework of ideas (O'Connell 2007), rather than focusing on one individual's reality. Previously published Cochrane reviews on couples therapy for depression (Barbato 2006) and family therapy for depression (Henken 2007) will be updated concurrently.

Studies of long-term, continuation or maintenance therapy interventions designed to prevent relapse of depression or to treat chronic depressive disorders will be excluded from the review. Similarly, studies of interventions designed to prevent a future episode of depression will be excluded.

Guided self-help, in which the practitioner provides brief face to face non-therapeutic support to patients who are using a self-help psychological therapy intervention, will be excluded as will bibliotherapy and writing therapies.

Psychological therapy that is provided wholly by telephone or over the Internet will not be eligible for inclusion.

Studies of dual modality treatments, in which patients are randomised to receive a behavioural therapy intervention combined with pharmacological treatment and compared with a treatment as usual control condition, will be excluded from the current review, and will be examined in a separate programme of reviews on combination treatments for depression.

Component or dismantling studies, in which the effectiveness of individual components of behavioural therapeutic approach are investigated, will not be included. Data from these studies will be extracted and included in a separate overview of psychological therapies for depression, in which multiple treatments meta-analysis (MTM) will be used to compare the relative effectiveness of all psychotherapies, regardless of whether they have been directly compared in direct RCTs. If there are sufficient data, we will use the MTM model proposed in Welton 2009 to allow conclusions to be drawn regarding which components, or combination of components, are most effective at reducing depressive symptoms. See Unit of analysis issues for further detail on MTM.

\section{Types of outcome measures}

\section{Primary outcomes}

1. Treatment efficacy: the number of patients who respond to treatment, based on changes on Beck Depression Inventory (BDI) (Beck 1961), Hamilton Rating Scale for Depression (HAM-D) (Hamilton 1960) or Montgomery-Asberg Depression Rating Scale (MADRS) (Montgomery 1979), or any other validated depression scale. Many studies define response by $50 \%$ or greater reduction on BDI, HAM-D etc. but some studies define response using Jacobson's Reliable Change Index; we will accept the study authors' original definition. If the original authors report several outcomes corresponding with our definition of response, we will give preference to BDI for self-rating scale and HAM-D for observer-rating scale.

2. Treatment acceptability: the number of participants who drop out of psychological therapy treatment for any reason. 


\section{Secondary outcomes}

1. The number of patients who remit on treatment, based on the endpoint absolute status of the patients, as measured by Beck Depression Inventory (BDI) (Beck 1961), Hamilton Rating Scale for Depression (HAM-D) (Hamilton 1960) or Montgomery-Asberg Depression Rating Scale (MADRS) (Montgomery 1979), or any other validated depression scale. Examples of definitions of remission include 10 or less on BDI, 7 or less on HAM-D or 10 or less on MADRS; we will accept the study authors' original definition. If the original authors report several outcomes corresponding with our definition of response, we will give preference to BDI for self-rating scale and HAM-D for observer-rating scale.

2. Improvement in depression symptoms, based on a continuous outcome of group mean scores at the end of treatment using BDI, HAM-D, MADRS, or any other validated depression scale.

3. Improvement in overall symptoms, using the Clinical Global Impressions scale (CGI) (Guy 1976).

4. Improvement in anxiety symptoms, measured using a validated continuous scale, either assessor-rated, such as the Hamilton Anxiety Scale [HAM-A] (Hamilton 1959) or self-report, including the Trait subscale of the Spielberger State-Trait Anxiety Inventory (STAI-T) (Spielberger 1983) and the Beck Anxiety Inventory (BAI) (Beck 1988).

5. Adverse effects, such as completed suicides, attempted suicides and worsening of symptoms, where reported, will be summarised in narrative form.

6. Social adjustment, social functioning including the Global Assessment of Function (Luborsky 1962) scores, where reported, will be summarised in narrative form.

7. Quality of life, using validated measures such as the SF-36 (Ware 1993), HoNOS (Wing 1994) and WHOQOL (WHOQOL 1998), where reported, will be summarised in narrative form.

8. Economic outcomes (e.g. days of work absence/ability to return to work, number of appointments with primary care physician, number of referrals to secondary services, use of additional treatments) where reported, will be summarised in narrative form.

\section{Search methods for identification of studies \\ Electronic searches}

CCDANCTR Registers: We will search two clinical trials registers created and maintained by the Cochrane Depression, Anxiety and Neurosis Group (CCDAN), the CCDANCTRStudies Register and the CCDANCTR-References Register. References to trials for inclusion in the Group's registers are collated from routine (weekly) searches of MEDLINE, EMBASE and PsycINFO, quarterly searches of the Cochrane Central Register of Controlled Trials (CENTRAL) and additional ad hoc searches of other databases (PSYNDEX, 
LILACS, AMED, CINAHL). These searches employ generic terms for depression anxiety and neuroses; together with sensitive (database specific) RCT filters.

References to trials are also sourced from international trials registers via the World Health Organisation's trials portal (http://apps.who.int/trialsearch/); drug companies; the handsearching of key journals, conference proceedings and other (non-Cochrane) systematic reviews and meta-analyses.

Details of the generic search strategies can be found in the 'Specialized Register' section of the Cochrane Depression, Anxiety and Neurosis Group's module text.

1. The CCDANCTR-Studies Register: The CCDANCTR-Studies Register contains over 11,000 trials for the treatment or prevention of depression, anxiety and neurosis. Each trial has been coded using the EU-Psi coding manual (as a guide) and includes information on intervention, condition, comorbidities, age, treatment setting etc.

The studies register will be searched using the following search terms:

Condition $=($ depress $*$ or dysthymi $*)$ and Intervention $=(*$ therap $*$ or training $)$

2. The CCDANCTR-References Register: The CCDANCTR-References Register contains bibliographic records of reports of trials coded in the CCDANCTR-Studies Register together with several other uncoded references (total number of records $=24,500$ ). This register will be searched using a comprehensive list of terms for 'psychotherapies' as indicated in Appendix 1. Records already retrieved from the search of the CCDANCTR-Studies Register will be de-duplicated.

\section{Searching other resources}

1. Reference lists: The references of all selected studies will be searched for more published reports and citations of unpublished studies. Relevant review papers will be checked.

2. Personal communication: Subject experts will be contacted to check that all relevant studies, either published or unpublished, have been considered for inclusion.

\section{Data collection and analysis}

Selection of studies-Two review authors (RC and $\mathrm{VH})$ will examine the abstracts of all publications obtained through the search strategy. Full articles of all the studies identified by either of the review authors will then be obtained and inspected by the same two review authors for trials meeting the following criteria:

1. Randomised controlled trial;

2. Participants have depression diagnosed by operationalised criteria;

3. Any BT approach (behavioural therapy, social skills training, behavioural activation, relaxation training) compared with non-treatment, waiting list control or treatment as usual. Conflicts of opinion regarding eligibility of a study will be discussed with a third review author, having retrieved the full paper and consulted 
the authors if necessary, until consensus is reached. External subject or methodological experts will be consulted if necessary.

Data extraction and management-Data will be extracted by two review authors, with data from each study being extracted independently by two of these authors. Any disagreement will be discussed with an additional review author and where necessary, the authors of the studies will be contacted for further information.

Information relating to study population, sample size, interventions, comparators, potential biases in the conduct of the trial, outcomes including adverse events, follow-up and methods of statistical analysis will be abstracted from the original reports into specially designed paper forms then entered into a spreadsheet..

Management of time points: Post-treatment outcomes and outcomes at each reported follow-up point will be summarised. Where appropriate and if the data allow, outcomes will be categorised as short term (up to 6 months post-treatment), medium term ( 7 to12 months post-treatment) and long term (longer than 12 months).

Waiting list controls: A commonly used 'treatment as usual' is to randomise participants to active intervention groups and control group, and then to provide the active intervention to both groups but to delay delivery of the intervention to the control group until after those in the intervention group have completed treatment. Thus both groups receive the active intervention but at different times. Sometimes trialists describe this as a 'Waiting list control' or the control participants are placed 'on a waiting list'.

Data are collected at baseline, when groups are randomised and at the 'end-of-treatment' at which point participants in the active intervention group stop receiving the active intervention and the participants on the waiting list start the active intervention. There may be a follow-up of participants at time points after the end-of-treatment.

For studies like this we will include data up to the time point at which the waiting list participants start to receive treatment. Follow-up data for these participants will not be used. To include follow-up data could introduce bias as the intervention is not as originally allocated at the point of randomisation and participants may no longer be comparable. For instance, baseline risk for participants who received delayed treatment may have changed (e.g. participants may be more depressed).

Assessment of risk of bias in included studies-Risk of bias will be assessed for each included study using the Cochrane Collaboration 'risk of bias' tool (Higgins 2008). The following six domains will be considered:

1. Sequence generation: was the allocation sequence adequately generated?

2. Allocation concealment: was allocation adequately concealed?

3. Blinding of participants, personnel and outcome assessors for each main outcome or class of outcomes: was knowledge of the allocated treatment adequately prevented during the study? 
4. Incomplete outcome data for each main outcome or class of outcomes: were incomplete outcome data adequately addressed?

5. Selective outcome reporting: are reports of the study free of suggestion of selective outcome reporting?

6. Other sources of bias: was the study apparently free of other problems that could put it at a high risk of bias? Additional items to be included here are therapist qualifications, treatment fidelity and researcher allegiance/conflict of interest.

A description of what was reported to have happened in each study will be provided, and a judgement on the risk of bias will be made for each domain within and across studies, based on the following three categories:
A. Yes (low risk of bias)
B. Unclear
C. No (high risk of bias).

Two independent review authors will assess the risk of bias in selected studies. Any disagreement will be discussed with a third review author. Where necessary, the authors of the studies will be contacted for further information. All risk of bias data will be presented graphically and described in the text. Allocation concealment will be used as a marker of trial quality for the purposes of undertaking sensitivity analyses.

\section{Measures of treatment effect}

Continuous outcomes: Where studies have used the same outcome measure for comparison, data will be pooled by calculating the mean difference (MD). Where different measures are used to assess the same outcome, data will be pooled with standardised mean difference (SMD) and 95\% confidence intervals calculated.

Dichotomous outcomes: These outcomes will be analysed by calculating a pooled odds ratio (OR) and 95\% confidence intervals for each comparison. Because ORs can be difficult to interpret, these pooled ORs will be converted to relative risks (RR) using the formula provided in The Cochrane Handbook (Higgins 2008) and presented in this form for ease of interpretation.

Unit of analysis issues-Studies with more than two intervention arms can pose analytical problems in pair-wise meta-analysis. Where studies have two or more active treatment arms to be compared against treatment as usual, data will be managed in this review as follows:

Continuous data: Means, SDs and number of participants for each active treatment group will be pooled across treatment arms as a function of the number of participants in each arm to be compared against the control group (Law 2003; Higgins 2008; Higgins 2008a). 
Dichotomous data: Active treatment groups will be collapsed into a single arm for comparison against the control group, or the control group will be split equally between the treatment groups.

Multiple treatment meta-analysis: One method which retains the individual identity of each intervention and allows multiple intervention comparisons to be made, without the need to lump or split intervention arms, is a multiple treatment meta-analysis (MTM) (Lu 2004; Caldwell 2005; Cipriani 2009b). MTM (also known as Mixed Treatment Comparison or Network Meta-analysis) refers to ensembles of trial evidence in which direct and indirect evidence on relative treatment effects are pooled. The objective of an MTM analysis is to combine all the available trial evidence into an internally consistent set of estimates while respecting the randomisation in the evidence. An MTM provides estimates of the effect of each intervention relative to every other, whether or not they have been directly compared in trials. One can also calculate the probability that each treatment is the most effective. We do not intend to use an MTM in this review, as we are unlikely to have sufficient data for the analysis. However, this review forms part of a series of 12 reviews which will contribute studies to an overview of reviews (Higgins 2008a; Becker 2008) in which MTM will be used as the main analytical strategy.

Dealing with missing data-Missing dichotomous data will be managed through intention to treat (ITT) analysis, in which it will be assumed that patients who dropped out after randomisation had a negative outcome. Best / worse case scenarios will also be calculated for the clinical response outcome, in which it will be assumed that dropouts in the active treatment group had positive outcomes and those in the control group had negative outcomes (best case scenario), and that dropouts in the active treatment group had negative outcomes and those in the control group had positive outcomes (worst case scenario), thus providing boundaries for the observed treatment effect. If there is a large amount of missing information then these best / worst case scenarios will be given greater emphasis in the presentation of the results.

Missing continuous data will either be analysed on an endpoint basis, including only participants with a final assessment, or analysed using last observation carried forward to the final assessment (LOCF) if LOCF data were reported by the trial authors. Where SDs are missing, attempts will be made to obtain these data through contacting trial authors. Where SDs are not available from trial authors, they will be calculated from P values, t-values, confidence intervals or standard errors, where reported in articles (Deeks 1997).

Where the vast majority of actual SDs are available and only a minority of SDs are unavailable or unobtainable, a method used for imputing SDs and calculating percentage responders devised by Furukawa and colleagues (Furukawa 2005; Furukawa 2006) will be used. Where this method is employed, data will be interpreted with caution, taking account of the degree of heterogeneity observed. A sensitivity analysis will also be undertaken to examine the effect of the decision to use imputed data. 
Where additional figures are not available or obtainable, and it is not deemed appropriate to use the Furukawa method described above, the study data will not be included in the comparison of interest.

Assessment of heterogeneity-Statistical heterogeneity will be formally tested using the $\mathrm{chi}^{2}$ test, which provides evidence of variation in effect estimates beyond that of chance. Since the $\mathrm{chi}^{2}$ test has low power to assess heterogeneity where a small number of participants or trials are included, the $P$ value will be conservatively set at 0.1 . Heterogeneity will also be quantified using the $\mathrm{I}^{2}$ statistic, which calculates the percentage of variability due to heterogeneity rather than chance. We expect, a priori, that there will be considerable clinical heterogeneity between studies and so $\mathrm{I}^{2}$ values in the range of $50 \%$ to $90 \%$ will be considered to represent substantial statistical heterogeneity and will be explored further. However, the importance of the observed $\mathrm{I}^{2}$ will depend on the magnitude and direction of treatment effects and the strength of evidence for heterogeneity (Higgins 2003; Deeks 2008). Forest plots generated in RevMan 5 now also provide an estimate of tau $^{2}$, the between-study variance in a random-effects meta-analysis. To give an indication of the spread of true intervention effects we will use the tau ${ }^{2}$ estimate to form an approximate range of intervention effects using the method outlined in section 9.5.4 of the Cochrane Handbook for Systematic Reviews of Interventions (Deeks 2008). This will be undertaken for the primary outcomes only.

Assessment of reporting biases-As far as possible, the impact of reporting biases will be minimised by undertaking comprehensive searches of multiple sources (including trial registries), increasing efforts to identify unpublished material, and including nonEnglish language publications.

We will also try and identify outcome reporting bias in trials by recording all trial outcomes, planned and reported and noting where there are missing outcomes. Where we find evidence of missing outcomes, we will attempt to obtain any available data direct from the authors.

Where sufficient numbers of trials allow for a meaningful analysis, funnel plots will be constructed to establish the potential influence of reporting biases and small study effects.

Data synthesis-Given the potential heterogeneity of psychological therapy approaches for inclusion, together with the likelihood of differing secondary comorbid mental disorders in the population of interest, a random-effects model will be used in all analyses.

\section{Subgroup analysis and investigation of heterogeneity Clinical heterogeneity}

1. Baseline depression severity: the severity of depression on entering the trial is expected to have an impact on outcomes. Heterogeneity analyses will categorise baseline severity as mild, moderate or severe.

2. Number of sessions: there are likely to be differences in the numbers of therapy sessions received and this is expected to affect treatment outcome. Numbers of 
sessions will be categorised as 1 to 7 sessions, 8 to 12 sessions, 13 to 20 sessions and more than 20 sessions.

3. Type of comparison: the type of comparator used is likely to influence the observed effectiveness of the intervention. Where possible, comparators will be categorised as waiting list or treatment as usual/usual care or attention placebo.

4. Strength of therapeutic alliance/perceived therapist empathy, based on validated measures such as the Barrett-Lennard Relationship Inventory (Barrett-Lennard 1986) or Working Alliance Inventory (Horvath 1986): where reported, this information will be summarised in narrative form.

\section{Sensitivity analysis}

1. Fidelity to treatment: studies that have not assessed fidelity to the psychological therapy model(s) under evaluation through assessment of audio or videotapes of therapy sessions will be excluded.

2. Study quality: allocation concealment will be used as a marker of trial quality. Studies that have not used allocation concealment will be excluded.

3. Trials where missing data have been imputed will be excluded.

4. Antidepressant treatment (naturalistic use; combination treatment used in both psychological therapy arms).

5. Trials included in the review following post-hoc decisions about their eligibility as behavioural therapeutic approaches will be excluded.

\section{Acknowledgments}

Internal sources

- University of Bristol, UK.

External sources

- $\quad$ NIHR Programme Grant - UK Department of Health, UK.

\section{WHAT'S NEW}

\begin{tabular}{lll}
\hline Date & Event & Description \\
\hline 1 June 2012 & Amended & Minor changes to methods \\
\hline
\end{tabular}

\section{HISTORY}

Protocol first published: Issue 9, 2010

\section{Appendix 1. CCDAN-CTR References Register search (psychotherapies for depression)}

Title, Abstract, Keywords $=($ depress* or dysthymi* $)$ 
and

Title, Abstract, Keywords = $((*$ therap* and $(($ acceptance* or commitment* $)$ or "activity scheduling" or alderian or art or aversion or brief or "client cent*" or cognitive or color or colour or "compassion-focused" or "compassion* focus*" or compassionate or conjoint or conversion or conversational or couples or dance or dialectic* or diffusion or distraction or eclectic or "emotion* focus*" or emotion-focus* or existential or experiential or exposure or expressive or family or focus-oriented or "focus oriented" or freudian or gestalt or group or humanistic or implosive or insight or integrative or interpersonal or jungian or kleinian or marital or metacognitive or meta-cognitive or milieu or morita or multimodal or multimodal or music or narrative or nondirective or non-directive or "non directive" or nonspecific or non-specific or "non specific" or "object relations" or "personal construct" or "person cent*" or person-cent* or persuasion or play or "pleas* event*" or primal or problem-focused or "problem focused" or problem-solving or "problem solving" or processexperiential or "process experiential" or psychodynamic or "rational emotive" or reality or "reciprocal inhibition" or relationship* or reminiscence or restructuring or rogerian or schema* or self-control* or "self control*" or "short term" or short-term or sex or "social effectiveness" or "social skill*" or socio-environment* or "socio environment*" or "solution focused" or solution-focused or "stress management" or supportive or time-limited or "time limited" or "third wave" or transference or transtheoretical or validation)) or abreaction or "acting out" or "age regression" or ((assertive* or autogenic or mind or sensitivity) and train*) or autosuggestion or "balint group" or ((behavior* or behaviour*) and (activation or therap* or treatment or contracting or modification)) or biofeedback or catharsis or cognitive or "mind training" or counsel* or "contingency management" or countertransference or "covert sensitization" or "eye movement desensiti*" or "crisis intervention" or "dream analysis" or "emotional freedom" or "free association" or "functional analys*" or griefwork or "guided imagery" or hypno* or imagery or meditation* or "mental healing" or mindfulness* or psychoanaly* or psychodrama or psychoeducat* or "psycho* support*" or psychotherap* or relaxation or "role play*" or "self analysis" or "self esteem" or "sensitivity training" or "support* group*" or therapist or "therapeutic technique*" or "transactional analysis")

\section{Additional references}

APA 1980 . American Psychiatric Association. Diagnostical and Statistical Manual of Mental Disorders (DSM-III). 3rd Edition. American Psychiatric Association; Washington DC: 1980.

APA 1987 . American Psychiatric Association. Diagnostical and Statistical Manual of Mental Disorders (DSM-III-R). 3rd Edition. American Psychiatric Association; Washington DC: 1987.

APA 1994 . American Psychiatric Association. Diagnostical and Statistical Manual of Mental Disorders (DSM-IV). 4th Edition. American Psychiatric Association; Washington DC: 1994.

APA 2000 . American Psychiatric Association. Diagnostical and Statistical Manual of Mental Disorders (DSM-IV-TR). 4th Edition. American Psychiatric Association; Washington DC: 2000.

Arroll 2009 . Arroll B, Elley CR, Fishman T, Goodyear-Smith FA, Kenealy T, Blashki G, et al. Antidepressants versus placebo for depression in primary care. Cochrane Database of Systematic Reviews. 2009; 3 DOI: 10.1002/14651858.CD007954.

Barbato 2006 . Barbato A, D’Avanzo B. Marital therapy for depression. Cochrane Database of Systematic Reviews. 2006; (2) DOI: 10.1002/14651858.CD004188.pub2. 
Barrett-Lennard 1986 . Barrett-Lennard, GT. The relationship inventory now: Issues and advances in theory, method and use. The Psychotherapeutic Process: A Research Handbook. Greenberg, LS.; Pinsof, W., editors. Guildford Press; New York: 1986.

Bayer 2000 . Bayer A, Tadd W. Unjustified exclusion of elderly people from studies submitted to research ethics committee for approval: descriptive study. BMJ. 2000; 321(7267):992-3. [PubMed: 11039965]

Beck 1961 . Beck AT, Ward CH, Mendelsohn M, Mock J, Erbaugh J. An inventory for measuring depression. Archives of General Psychiatry. 1961; 4:561-71. [PubMed: 13688369]

Beck 1979 . Beck, AT.; Rush, AJ.; Shaw, BF.; Emery, G. Cognitive Therapy of Depression. Guildford Press; New York: 1979.

Beck 1988 . Beck AT, Epstein N, Brown G, Steer RA. An inventory for measuring clinical anxiety: psychometric properties. Journal of Consulting and Clinical Psychology. 1988; 56(6):893-7. [PubMed: 3204199]

Becker 2008 . Becker, LA.; Oxman, AD.; Higgins, JPT. Overviews of reviews. In: Green, S., editor. Cochrane Handbook for Systematic Reviews of Interventions Version 5.0.2 [updated September 2009]. The Cochrane Collaboration; 2008.

Bellack 1980 . Bellack AS, Hersen M, Himmelhock JM. Social skills training for depression: A treatment manual. JSAS Cat Select Doc. 1980; 10(92 (Ms No 21964):25.

Bernstein 1973 . Bernstein, DA.; Borkovec, TD. Progressive Relaxation Training. Research Press; Champaign, Illinois: 1973.

Caldwell 2005 . Caldwell DM, Ades AE, Higgins JPT. Simultaneous comparison of multiple treatments: combining direct and indirect evidence. BMJ. 2005; 331(7521):897-900. [PubMed: 16223826]

Chen 2001 . Chen YF, Dewey ME, Avery AJ. Self-reported medication use for older people in England and Wales. Journal of Clinical Pharmacy and Therapeutics. 2001; 26(2):129-40. [PubMed: 11350536]

Churchill 2000 . Churchill R, Khaira M, Gretton V, Chilvers C, Dewey M, Duggan C, et al. Treating depression in general practice: factors affecting patients' treatment preferences. British Journal of General Practice. 2000; 50(460):905-6. [PubMed: 11141877]

Churchill 2001 . Churchill R, Hunot V, Corney R, Knapp M, McGuire H, Tylee A, et al. A systematic review of controlled trials of the effectiveness and cost-effectiveness of brief psychological treatments for depression. Health Technology Assessment. 2001; 5(35):1-173. [PubMed: 12387733]

Cipriani 2005 . Cipriani A, Brambilla P, Furukawa T, Geddes J, Gregis M, Hotopf M, et al. Fluoxetine versus other types of pharmacotherapy for depression. Cochrane Database of Systematic Reviews. 2005; (4) DOI: 10.1002/14651858.CD004185.pub2.

Cipriani 2009 . Cipriani A, Santilli C, Furukawa TA, Signoretti A, Nakagawa A, McGuire H, et al. Escitalopram versus other antidepressive agents for depression. Cochrane Database of Systematic Reviews. 2009; (2) DOI: 10.1002/14651858.CD006532.pub2.

Cipriani 2009a . Cipriani A, La Ferla T, Furukawa TA, Signoretti A, Nakagawa A, Churchill R, et al. Sertraline versus other antidepressive agents for depression. Cochrane Database of Systematic Reviews. 2009; (2) DOI: 10.1002/14651858.CD006117.pub4.

Cipriani 2009b . . Cipriani A, Furukawa TA, Salanti G, Geddes JR, Higgins JP, Churchill R, et al. Comparative efficacy and acceptability of 12 new-generation antidepressants: a multipletreatments meta-analysis. Lancet. 2009; 373(9665):746-58. [PubMed: 19185342]

Cuijpers 2002 . Cuijpers P, Smit F. Excess mortality in depression: a meta-analysis of community studies. Journal of Affective Disorders. 2002; 72:227-36. [PubMed: 12450639]

Cuijpers 2007 . Cuijpers P, van Straten A, Warmerdam L. Behavioral activation treatments of depression: a meta-analysis. Clinical Psychology Review. 2007; 27(3):318-26. [PubMed: 17184887]

Cuijpers 2008 . Cuijpers P, van Straten A, Andersson G, van Oppen P. Psychotherapy for depression in adults: a meta-analysis of comparative outcome studies. Journal of Consulting and Clinical Psychology. 2008; 76(6):909-22. [PubMed: 19045960] 
De Shazer 1988 . De Shazer, S. Clues: investigating solutions in brief therapy. W.W. Norton; New York: 1988.

Deeks 1997 . Deeks JJ. Are you sure that's a standard deviation? (part 1). Cochrane News. 1997; 10:11-2.

Deeks 2008 . Deeks, JJ.; Higgins, JPT.; Altman, DG.; On behalf of the Cochrane Statistical Methods Group. Analysing data and undertaking meta-analyses. In: Higgins, JPT.; Green, S., editors. Cochrane Handbook for Systematic Reviews of Interventions Version 5.0.2 [updated September 2009]. The Cochrane Collaboration; 2008.

Dimidjian 2006 . Dimidjian S. Randomized trial of behavioral activation, cognitive therapy, and antidepressant medication in the acute treatment of adults with major depression. Journal of Consulting and Clinical Psychology. 2006; 74(4):658-70. [PubMed: 16881773]

Ekers 2008 . Ekers D, Richards D, Gilbody S, Ekers D, Richards D, Gilbody S. A meta-analysis of randomized trials of behavioural treatment of depression. Psychological Medicine. 2008; 38(5): 611-23. [PubMed: 17903337]

Ellis 1962 . Ellis, A. Reason and emotion in psychotherapy. Lyle Stuart; New York: 1962.

Ellis 2004 . Ellis P. Australian and New Zealand clinical practice guidelines for the treatment of depression. Australian and New Zealand Journal of Psychiatry. 2004; 38(6):389-407. [PubMed: 15209830]

ESEMeD/MHEDEA 2004 . ESEMeD/MHEDEA 2000 investigators. Alonso J, Angermeyer MC, Bernert S, Bruffaerts R, Brugha TS, et al. Prevalence of mental disorders in Europe: results from the European Study of the Epidemiology of Mental Disorders (ESEMeD) project. Acta Psychiatrica Scandinavica Suppl. 2004; 190(420):21-7.

Eysenck 1960 . Eysenck, HJ. Behavior Therapy and the Neuroses. Pergamon; Oxford: 1960.

Freud 1949 . Freud, S. An outline of psychoanalysis. Hogarth Press; London: 1949.

Fuchs 1977 . Fuchs CZ, Rehm LP. Self-Control Behavior-Therapy Program for Depression. Journal of Consulting and Clinical Psychology. 1977; 45(2):206-15. [PubMed: 850005]

Furukawa 2005 . Furukawa TA, Cipriani A, Barbui C, Brambilla P, Watanabe N. Imputing response rates from means and standard deviations in meta-analysis. Internal Clinical Psychopharmacology. 2005; 20:49-52.

Furukawa 2006 . Furukawa TA, Barbui C, Cipriani A, Brambilla P, Watanabe N. Imputing missing standard deviations in meta-analyses can provide accurate results. Journal of Clinical Epidemiology. 2006; 59:7-10. [PubMed: 16360555]

Guaiana 2007 . Guaiana G, Barbui C, Hotopf M. Amitriptyline for depression. Cochrane Database of Systematic Reviews. 2007; (3) DOI: 10.1002/14651858.CD004186.pub2.

Guy 1976 . Guy, W. ECDEU assessment manual for psychopharmacology. Division of Extramural Research Programs; NIMH Psychopharmacology Research Branch: 1976. p. 218-22.

Hamilton 1959 . Hamilton M. The assessment of anxiety states by rating. British Journal of Medical Psychology. 1959; 32(1):50-5. [PubMed: 13638508]

Hamilton 1960 . Hamilton M. A rating scale for depression. Journal of Neurology, Neurosurgery, and Psychiatry. 1960; 23:56-62.

Henken 2007 . Henken HT, Huibers MJ, Churchill R, Restifo K, Roelofs J. Family therapy for depression. Cochrane Database of Systematic Reviews. 2007; (3) DOI: 10.1002/14651858.CD006728.

Higgins 2003 . Higgins JPT, Thompson SG, Deeks JJ, Altman DG. Measuring inconsistency in meta-analyses. BMJ. 2003; 327(7414):557-60. [PubMed: 12958120]

Higgins 2008 . Higgins, JPT.; Green, S. Cochrane Handbook for Systematic Reviews of Interventions Version 5.0.2 [updated September 2009]. The Cochrane Collaboration; 2008. Available from www.cochrane-handbook.org

Higgins 2008a . Higgins, JPT.; Deeks, JJ.; Altman, DG.; On behalf of the Cochrane Statistical Methods Group. Special topics in statistics. In: Higgins, JPT.; Green, S., editors. Cochrane Handbook for Systematic Reviews of Interventions Version 5.0.2 [updated September 2009]. The Cochrane Collaboration; 2008. 
Hopko 2003 . Hopko DR, Lejuez CW, Ruggiero KJ, Eifert GH. Contemporary behavioral activation treatments for depression: Procedures, principles, and progress. Clinical Psychology Review. 2003; 23(5):699-717. [PubMed: 12971906]

Horvath 1986 . Horvath, AO.; Greenberg, L. The development of the Working Alliance Inventory: A research handbook. In: Greenberg, L.; Pinsoff, W., editors. Psychotherapeutic Processes: A Research Handbook. Guildford Press; New York: 1986.

Hunot 2007 . Hunot VM, Horne R, Leese MN, Churchill RC. A Cohort Study of Adherence to Antidepressants in Primary Care: The Influence of Antidepressant Concerns and Treatment Preferences. Primary Care Companion to the Journal of Clinical Psychiatry. 2007; 9(2):91-9.

Ivnik 1992 . Ivnik RJ, Malec JF, Smith GE, Tangalos EG, Petersen RC, Kokmen E, et al. Mayo's older americans normative studies: Updated AVLT norms for ages 56 to 97 . The Clinical Neuropsychologist. 1992; 6(1 supp 1):83-104.

Jackson 1985 . Jackson HJ, Moss JD, Solinski S. Social Skills Training - An Effective Treatment for Unipolar Nonpsychotic Depression. Australian and New Zealand Journal of Psychiatry. 1985; 19(4):342-53. [PubMed: 3914279]

Jacobson 1996 . Jacobson NS, Dobson KS, Truax PA, Addis ME, Koerner K, Gollan JK, et al. A component analysis of cognitive-behavioral treatment for depression. Journal of Consulting and Clinical Psychology. 1996; 64(2):295-304. [PubMed: 8871414]

Jacobson 2001 . Jacobson NS, Martell CR, Dimidjian S. Behavioral activation treatment for depression: Returning to contextual roots. Clinical Psychology-Science and Practice. 2001; 8(3): 255-70.

Jung 1963 . Jung, CG.; Jaffe, A.; Winston, C. Memories, dreams, reflections. Pantheon Books; New York: 1963.

Klein 1960 . Klein, M. Our Adult World and its Roots in Infancy. Tavistock; London: 1960.

Klerman 1984 . Klerman, GL.; Weissman, MM.; Rousaville, BJ.; Chevron, ES. Interpersonal psychotherapy for depression. Basic Books; New York: 1984.

Law 2003 . Law J, Garrett Z, Nye C. Speech and language therapy interventions for children with primary speech and language delay or disorder. Cochrane Database of Systematic Reviews. 2003; (3) DOI: 10.1002/14651858.CD004110.

Lazarus 1971 . Lazarus, AA. Behavior therapy and beyond. [S.1.]. McGraw; 1971.

Lewinsohn 1972 . Lewinsohn PM, Libet J. Pleasant Events, Activity Schedules, and Depressions. Journal of Abnormal Psychology. 1972; 79(3):291-5. [PubMed: 5033370]

Lewinsohn 1974 . Lewinsohn, PM. A Behavioural Approach to Depression. In: Friedman, RJ.; Katz, MM., editors. The psychology of depression: contemporary theory and research. Winston; Washington, D.C.: 1974. p. 157-78.

Lewinsohn 1984 . Lewinsohn, PM. The coping with depression course: a psychoeducational intervention for unipolar depression. Castalia Pub. Co.; Eugene, OR: 1984.

Lu 2004 . Lu G, Ades AE, Lu G, Ades AE. Combination of direct and indirect evidence in mixed treatment comparisons. Statistics in Medicine. 2004; 23(20):3105-24. [PubMed: 15449338]

Luborsky 1962 . Luborsky L. Clinician's judgments of mental health. Archives of General Psychiatry. 1962; 7:407-17. [PubMed: 13931376]

Martell 2001 . Martell, CR.; Addis, ME.; Jacobson, NS. Depression in context: strategies for guided action. London: W.W. Norton; New York: 2001.

Maslow 1943 . Maslow AH. A theory of human motivation. Psychological Review. 1943; 50:27096.

May 1961 . May, R. Existential psychology. Random House; New York: 1961.

McMurdo 2005 . McMurdo ME, Witham MD, Gillespie ND. Including older people in clinical research. BMJ. 2005; 331(7524):1036-7. [PubMed: 16269472]

Mitchell 2009 . Mitchell AJ, Vaze A, Rao S. Clinical diagnosis of depression in primary care: a meta-analysis. The Lancet. 2009; 374(9690):609-19.

Montgomery 1979 . Montgomery SA, Asberg M. A new depression scale designed to be sensitive to change. British Journal of Psychiatry. 1979; 134:382-9. [PubMed: 444788] 
Mykletun 2009 . Mykletun A, Bjerkeset O, Øverland S, Prince M, Dewey M, Stewart R. Levels of anxiety and depression as predictors of mortality: the HUNT study. British Journal of Psychiatry (2009). 2009; 195:118-125.

Nelson Jones 1990 . Nelson Jones, R. The Theory and Practice of Counselling Psychology. Cassell; London: 1990.

Nezu 1986 . Nezu AM. Efficacy of A Social Problem-Solving Therapy Approach for Unipolar Depression. Journal of Consulting and Clinical Psychology. 1986; 54(2):196-202. [PubMed: 3700806]

NICE 2009 . NICE. Clinical Guideline 09 and 91. National Institute for Clinical Excellence; London: 2009. Depression: Treatment management of depression in adults, including adults with a chronic physical health problem.

O’Connell 2007 . O’Connell, B. Solution-focused therapy. In: Dryden, W., editor. In Dryden's Handbook of Individual Therapy. Sage; London: 2007.

Pilgrim 2002 . Pilgrim, D. The Cultural Context of British Psychotherapy. In: Dryden, W., editor. The Handbook of Individual Psychotherapy. 4th Edition. Sage; London: 2002.

Rachman 1997 . Rachman, SJ. The evolution of cognitive behaviour therapy. In: Clark, DM.; Fairburn, CG., editors. Science and practice of cognitive behaviour therapy. Oxford University Press; Oxford: 1997. p. 2-26.

Rait 2005 . Rait G, Fletcher A, Smeeth L, Brayne C, Stirling S, Nunes M, et al. Prevalence of cognitive impairment: results from the MRC trial of assessment and management of older people in the community. Age Ageing. 2005; 34(3):242-8. [PubMed: 15863409]

Rait 2009 . Rait G, Walters K, Griffin M, Buszewicz M, Petersen I, Nazareth I. Recent trends in the incidence of recorded depression in primary care. The British Journal of Psychiatry. 2009; 195(6):520-4. [PubMed: 19949202]

Rehm 1977 . Rehm LP. Self-Control Model of Depression. Behavior Therapy. 1977; 8(5):787-804.

Riedel-Heller 2005 . Riedel-Heller SG, Matschinger H, Angermeyer MC. Mental disorders--who and what might help? Help-seeking and treatment preferences of the lay public. Social Psychiatry and Psychiatric Epidemiology. 2005; 40(2):167-74. [PubMed: 15685409]

Roca 2009 . Roca M, Gili M, Garcia-Garcia M, Salva J, Vives M, Garcia Campayo J, et al. Prevalence and comorbidity of common mental disorders in primary care. Journal of Affective Disorders. 2009; 119(1-3):52-8. [PubMed: 19361865]

Rogers 1951 . Rogers, C. Client-centered therapy: Its current practice, implications and theory. Constable; London: 1951.

Ryle 1990 . Ryle, A. Cognitive-analytic Therapy - Active Participation in Change: New Integration in Brief Psychotherapy. John Wiley \& Sons; Chichester: 1990.

Skinner 1953 . Skinner, BF. Science and human behaviour. Free Press; New York: 1953.

Spielberger 1983 . Spielberger, CD.; Gorsuch, RL.; Lushene, R.; Vagg, PR.; Jacobs, GA. Manual for the State-Trait Anxiety Inventory. Consulting Psychologists Press; Palo Alto, CA: 1983.

Stiles 2008 . Stiles WB, Barkham M, Mellor-Clark J, Connell J. Effectiveness of cognitivebehavioural, person-centred, and psychodynamic therapies in UK primary-care routine practice: replication in a larger sample. Psychological Medicine. 2008; 38(5):677-88. [PubMed: 17825124]

Ustun 2004 . Ustun TB, Ayuso-Mateos JL, Chatterji S, Mathers C, Murray CJ. Global burden of depressive disorders in the year 2000. British Journal of Psychiatry. 2004; 184:386-92. [PubMed: 15123501]

van Geffen 2009 . van Geffen EC, Gardarsdottir H, van Hulten R, van Dijk L, Egberts AC, Heerdink ER. Initiation of antidepressant therapy: do patients follow the GP's prescription? British Journal of General Practice. 2009; 59(559):81-7. [PubMed: 19192372]

Ware 1993 . Ware, JE.; Snow, KK.; Kosinski, M.; Gandek, B. SF-36 Health Survey: Manual and interpretation guide. Health Institute, New England Medical Center; Boston, MA: 1993.

Watanabe 2004 . Watanabe N, Churchill R, Hunot V, Furukawa TA. Psychotherapy for depression in children and adolescents (Protocol). Cochrane Database of Systematic Reviews. 2004; (4) DOI: 10.1002/14651858.CD005334.

Watson 1924 . Watson, JB. Behaviorism. Norton; New York: 1924. 
Watzlavick 1974 . Watzlavick, P.; Weakland, J.; Fisch, R. Change: principles of problem formation and problem resolution. W W Norton; New York: 1974. Brief Problem SolvingChange: principles of problem formation and problem resolution. New York: W W Norton

Welton 2009 . Welton NJ, Caldwell DM, Adamopoulos E, Vedhara K. Mixed treatment comparison meta-analysis of complex interventions: psychological interventions in coronary heart disease. American Journal of Epidemiology. 2009; 169(9):1158-65. [PubMed: 19258485]

WHO 1978 . WHO. The Ninth Revision of the International Classification of Diseases and Related Health Problems (ICD-9). World Health Organisation; Geneva: 1978.

WHO 1992 . WHO. The ICD-10 Classification of Mental and Behavioural Disorders. World Health Organisation; Geneva: 1992.

WHO 2001 . WHO. World Health Report 2001: Mental Health: New Understanding, New Hope. World Health Organisation; Geneva: 2001.

WHO 2004 . WHO. The global burden of disease: 2004 update. WHO; Geneva: 2004.

WHO 2008 .WHO. 2008. http://www.who.int/healthinfo/global_burden_disease/projections/en/ index.htmlhttp://www.who.int/healthinfo/global_burden_disease/projections/en/index.html

WHOQL 1998 . WHO. The World Health Organization Quality of Life Assessment (WHOQOL): development and general psychometric properties. Social Science and Medicine. 1998; 46:156985. 0277-9536 (Print), 0277-9536 (Linking), 12. [PubMed: 9672396]

Wilson 2008 . Wilson KC, Mottram PG, Vassilas CA. Psychotherapeutic treatments for older depressed people. Cochrane Database of Systematic Reviews. 2008; (1) DOI: 10.1002/14651858.CD004853.pub2.

Wing 1994 . Wing, J. Measuring mental health outcomes: a perspective from the Royal College of Psychiatrists. Outcomes into Clinical Practice. BMJ publishing; London: 1994.

Wolpe 1958 . Wolpe, J. Psychotherapy by Reciprocal Inhibition. Stanford University Press; Standford: 1958. Witwatersrand University Press; Johannesburg

*Indicates the major publication for the study 